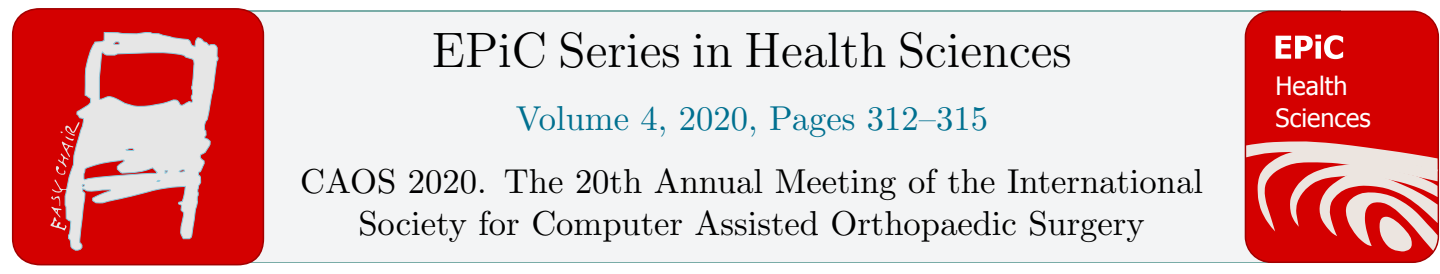

\title{
Fully Automatic Segmentation and Landmarking of Hip CT Images
}

\author{
Guoyan Zheng \\ Institute of Medical Robotics, School of Biomedical Engineering, Shanghai Jiao Tong University, \\ Shanghai, China \\ guoyan.zheng@sjtu.edu.cn
}

\begin{abstract}
We present a fully automatic method of segmenting and landmarking hip CT images for planning of Total Hip Arthroplasty (THA). Our method consists of two stages, i.e., the segmentation stage and the landmarking stage. At the segmentation stage, a multi-atlas segmentation constrained graph method is employed to fully automatically segment both the pelvis and the bilateral proximal femurs from the input CT data. The segmentation stage is followed by the landmarking stage, where a set of pre-defined landmarks are transferred from generic models of the associated hip structures to the input CT space via non-rigid registrations in order to compute a set of functional parameters that are relevant to planning of THA. Evaluated on 20 hip patients, we computed both the segmentation accuracy and the landmarking accuracy. An average segmentation error of $0.38 \pm 0.25$ $\mathrm{mm}$ and $0.49 \pm 0.22 \mathrm{~mm}$ was found for the hemi-pelvis and for the proximal femurs, respectively. For 3D landmarking, a mean error of $1.58 \pm 0.87 \mathrm{~mm}$ and $0.46 \pm 0.39 \mathrm{~mm}$ was found for the acetabular rim center and the acetabular rim radius, respectively; a mean error of $0.74 \pm 0.45^{\circ}$ was found for the orientation of the anterior pelvic plane; and a mean error of $3.14 \pm 1.90 \mathrm{~mm}$ and $2.04 \pm 1.61 \mathrm{~mm}$ was found for the femoral head center and the femoral offset, respectively.
\end{abstract}

\section{Introduction}

Landmark-based bone morphology and morphometric measurement is a well-established technique in surgical procedure requiring correct positioning of bones and prostheses. It provides various linear and angular measurements that are significant and useful for computer-aided diagnosis, computer-assisted surgery planning and evaluation, post-operative follow-up evaluation, and personalized prosthesis design. Most of existing computer-assisted diagnosis and planning systems are based on manual localization of landmarks that is not only time-consuming and requires a high level of expertise but also prone to intra- and inter-observer variations. There are several advantages of automatic landmarking approaches over manual analysis including faster analysis to save time for the surgeon, more standardized procedure to eliminate observer variability and more reliable measurements for improved clinical outcome comparison and data exchange.

F. Rodriguez Y Baena and F. Tatti (eds.), CAOS 2020 (EPiC Series in Health Sciences, vol. 4), pp. 312-315 
In this paper, we address the challenging problem of automatic 3D landmarking of hip CT images for Total Hip Arthroplasty (THA), which does not require any operator supervision or manual editing. Previous attempts for automatic landmarking from hip CT images usually assume that virtual bone models have already been extracted from the CT data, which is usually done with manual segmentation or semi-automatic segmentation $[1,2,3,5]$. The disadvantage of manual segmentation is that it is time consuming and the results are not really reproducible because the image interpretations by humans may vary significantly across interpreters.

\section{Materials and Methods}

\subsection{Methods}

Our method consists of two stages i.e., the segmentation stage and the landmarking stage. At the segmentation stage, a multi-atlas segmentation constrained graph method [4] is employed to fully automatically segment both the pelvis and the bilateral proximal femurs from the input CT data. The segmentation stage is followed by the landmarking stage, where a set of predefined landmarks are transferred from generic models of the associated hip structures to the input CT space via non-rigid registrations in order to compute a set of functional parameters that are relevant to planning of THA.

More specifically, starting from random forest regression-based organ detection, our segmentation method uses multi-atlas based mesh fusion results to initialize a bone sheetness based multi-label graph cut for an accurate hip CT segmentation which has the inherent advantage of automatic separation of the pelvic region from the bilateral proximal femoral regions. We then introduce graph cut constrained graph search algorithm to further improve the segmentation accuracy around the bilateral hip joint regions.

Additionally, we compute mean models of the pelvis and the bilateral proximal femurs by establishing correspondences of the associated hip structures across all atlases. We further define a set of anatomical landmarks on those mean models as shown in Figure 1. Given any target hip CT image, after automatic segmentation using the method described above, at the landmarking stage we conduct non-rigid registrations between the segmented binary volumes of hip structures and the binary volumes of the associated mean models to transfer the set of pre-defined landmarks from the associated mean model space to the target hip CT space. We use the open source framework "Elastix" [6] to conduct the non-rigid registrations. After that, we can use those transferred landmarks to compute a set of important clinical parameters. Specifically, as shown in Figure 1-(A), we first fit a plane from the transferred acetabular rim points to compute the normal of the acetabulum rim plane (NARP). We then conduct a 3D circle fitting of the transferred acetabular rim point to compute the acetabular rim center (ARC) as well as the acetabular rim radius (ARR). We also compute the normal of the anterior pelvic plane (NARP). All these parameters are clinically important for computer-assisted planning of the acetabular cup. Additionally, the transferred femoral landmarks as shown in Figure 1-(B) allow us to compute the femoral head center (FHC), the femoral offset (FO), the femoral shaft axis (FSA), the femoral neck axis (FNA) and the neck-shaft anlge (NSA). All these parameters are clinically important for computer-assisted planning of femoral stem.

\subsection{Experimental Design}

In this study, we take 30 hip CT images (60 hips) with the associated manual segmentation as the atlases. We then evaluated our method on another 20 hip CT images with ground truth 


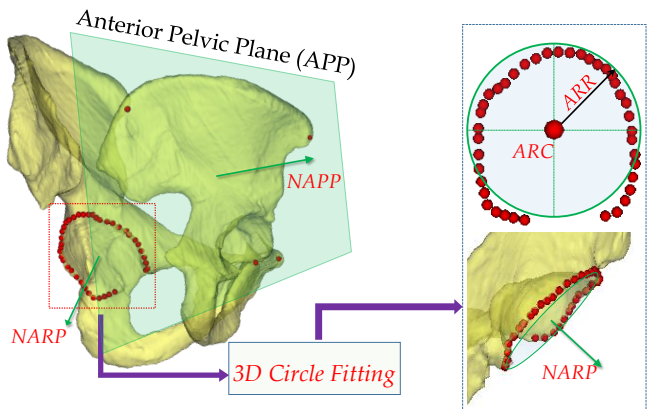

(A)

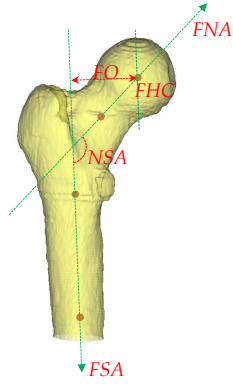

(B)
Pelvic Parameters

Acetabular Rim Center (ARC)

Acetabular Rim Radius (ARR)

Normal of the Acetabulum Rim Plane (NARP) Normal of the Anterior Pelvic Plane (NAPP)

Femoral Parameters

Femoral Header Center (FHC)

Femoral Offset (FO)

Femoral Shaft Axis (FSA)

Femoral Neck Axis (FNA)

Neck-Shaft Angle (NSA)

(C)

Figure 1: A schematic view of the set of landmarks, shown as red spheres pre-defined on the mean pelvic model and on the mean proximal femur model, as well as important clinical parameters that are computed from the set of pre-defined landmarks. (A) the set of landmarks pre-defined on the mean pelvic model and the associated clinical parameters; (B) the set of landmarks pre-defined on the mean proximal femur model and the associated clinical parameters; $(\mathrm{C})$ the list of important pelvic parameters and femoral parameters that are computed from the pre-defined landmarks.

Table 1: Automatic landmarking accuracy when evaluated on 20 hip CT images.

\begin{tabular}{|l|l|l|l|l|l|l|l|}
\cline { 2 - 7 } \multicolumn{1}{c|}{} & \multicolumn{4}{c|}{ Pelvic Parameters } & \multicolumn{3}{c|}{ Femoral Parameters } \\
\cline { 2 - 8 } \multicolumn{1}{c|}{} & ARC $(\mathrm{mm})$ & ARR $(\mathrm{mm})$ & ${\text { NARP }\left({ }^{\circ}\right)}$ & NAPP $\left(^{\circ}\right)$ & FHC $(\mathrm{mm})$ & FO $(\mathrm{mm})$ & NSA $\left(^{\circ}\right)$ \\
\hline Mean & $1.58 \pm 0.87$ & $0.46 \pm 0.39$ & $2.74 \pm 2.0$ & $0.74 \pm 0.45$ & $3.14 \pm 1.90$ & $2.04 \pm 1.61$ & $3.54 \pm 3.0$ \\
\hline Median & 1.29 & 0.34 & 2.25 & 0.70 & 2.95 & 1.73 & 2.25 \\
\hline
\end{tabular}

segmentation and anatomical landmarks that were obtained by manual analysis. For each data, one side was used. There were 11 left hips and 9 right hips. We evaluated both automatic segmentation accuracy as well as automatic landmarking accuracy. For segmentation, we computed the Dice overlap coefficient (Dice) as well as the average symmetric surface distance (ASSD) between the automatic segmentation and the manual segmentation. For landmarking, we evaluated errors on defining linear and angular parameters between those computed from the automatic landmarking and those computed from the manual landmarking.

\section{Results}

For segmentation, our approach achieved a mean Dice overlap coefficient of $96.95 \pm 1.96 \%$ and $96.92 \pm 1.42 \%$ for the hemi-pelvis and for the proximal femurs, respectively. A mean ASSD of $0.38 \pm 0.25 \mathrm{~mm}$ and $0.49 \pm 0.22 \mathrm{~mm}$ was found for the hemi-pelvis and for the proximal femurs, respectively.

Automatic landmarking results are shown in Table 1, where the results on defining the pelvic parameters as well as the femoral parameters are presented. Our approach achieved a mean error of $1.58 \pm 0.87 \mathrm{~mm}, 0.46 \pm 0.39 \mathrm{~mm}, 2.74 \pm 2.0^{\circ}, 0.74 \pm 0.45^{\circ}, 3.14 \pm 1.90 \mathrm{~mm}, 2.04 \pm 1.61 \mathrm{~mm}$ and $3.54 \pm 3.0^{\circ}$ for ARC, ARR, NARP, NAPP, FHC, FO, and NSA, respectively. 


\section{Discussions}

The results achieved by our method are comparable to those by the state-of-the-art methods, though direct comparison of different methods is difficult as not all of them are evaluated on the same dataset. For example, Fischer et al. [5] introduced a robust method for automatic identification of landmarks on surface models of the pelvis and they reported median differences below $3 \mathrm{~mm}$ for the position of the landmarks and below $1^{\circ}$ for the orientation of the anterior pelvic plane (APP) coordinate system. In this study, we achieved a median difference of $0.70^{\circ}$ for the orientation of the APP. Our approach has the additional advantage of automatically segmenting a given hip CT image $[1,2,3,5]$, which is important for computer-assisted planning of total hip arthroplasty. Our future work will be on extending the developed technique for computer-assisted planning of total knee arthroplasty (TKA).

\section{References}

[1] P Cerveri, M Marchente, W Bartels, K Corten, JP Simon, and A. Manzotti. Fautomated method for computing the morphological and clinical parameters of the proximal femur using heuristic modeling techniques. Ann Biomed Eng, 38(5):1752-1766, 2010.

[2] P Cerveri, M Marchente, C Chemello, N Confalonieri, A Manzotti, and G. Baroni. Advanced computational framework for the automatic analysis of the acetabular morphology from the pelvic bone surface for hip arthroplasty applications. Ann Biomed Eng, 39:2791-2806, 2011.

[3] X Chen, P Jia, Y Wang, H Zhang, L Wang, AF Frangi, and ZA Taylor. A surface-based approach to determine key spatial parameters of the acetabulum in a standardized pelvic coordinate system. Med Eng Phys, 52:22-30, 2018.

[4] C Chu, J Bai, X Wu, and G Zheng. Mascg: Multi-atlas segmentation constrained graph method for accurate segmentation of hip ct images. Med Image Anal., 26(1):173-184, 2015.

[5] MCM Fischer, F Krooss, J Habor, and K Radermacher. A robust method for automatic identification of landmarks on surface models of the pelvis. Sci Rep, 9(1):13322, 2019.

[6] S Klein, M Staring, K Murphy, MA Viergever, and JP. Pluim. elastix: a toolbox for intensity-based medical image registration. IEEE Trans Med Imaging, 29(1):196-205, 2010. 